\title{
A new EFT approach to NN scattering problem
}

\author{
E. Epelbaum • J. Gegelia
}

Received: date / Accepted: date

\begin{abstract}
We present the new, modified Weinberg approach to the NN scattering problem in effective field theory. Issues of renormalization are briefly discussed and the results of LO calculations are presented.
\end{abstract}

Keywords Effective field theory $\cdot$ Nucleon-nucleon scattering $\cdot$ Renormalization

\section{Introduction}

The few-nucleon sector of baryon chiral perturbation theory was first considered in Ref. [1] (For a recent review see e.g. 22). Within Weinberg's approach, the nucleonnucleon $(\mathrm{NN})$ potential is defined as a sum of all two-nucleon-irreducible time ordered diagrams of the non-relativistic effective field theory (EFT). It is calculated as a systematic expansion in small parameters. A finite number of diagrams contribute in the effective potential up to any finite order. The scattering amplitude is obtained by solving the Lippmann-Schwinger (LS) equation

$$
T\left(\mathbf{p}^{\prime}, \mathbf{p}, k\right)=V\left(\mathbf{p}^{\prime}, \mathbf{p}\right)+\hbar m \int \frac{d^{3} \mathbf{q}}{(2 \pi)^{3}} V\left(\mathbf{p}^{\prime}, \mathbf{q}\right) \frac{1}{\mathbf{p}^{2}-\mathbf{q}^{2}+i \epsilon} T(\mathbf{q}, \mathbf{p}, k),
$$

Presented at the 20th International IUPAP Conference on Few-Body Problems in Physics, 20 - 25 August, 2012, Fukuoka, Japan

E. Epelbaum

Institut für Theoretische Physik II, Fakultät für Physik und Astronomie, Ruhr-Universität Bochum 44780 Bochum, Germany

Tel.: +49 (0)234 32-23707

Fax: +49 (0)234 32-14697

E-mail: evgeny.epelbaum@ruhr-uni-bochum.de

J. Gegelia

Institut für Theoretische Physik II, Fakultät für Physik und Astronomie,

Ruhr-Universität Bochum 44780 Bochum, Germany,

Tbilisi State University, 0186 Tbilisi, Georgia

Tel.: +49 (0)234 32-28957

Fax: +49 (0)234 32-14697

E-mail: Jambul.Gegelia@tp2.ruhr-uni-bochum.de 
where $\hbar$ is included to keep the trace of the loop integration. The leading order NN potential is given by

$$
V_{0}=C_{S}+C_{T} \boldsymbol{\sigma}_{1} \cdot \boldsymbol{\sigma}_{2}-\frac{g_{A}^{2}}{4 F^{2}} \boldsymbol{\tau}_{1} \cdot \boldsymbol{\tau}_{2} \frac{\boldsymbol{\sigma}_{1} \cdot \mathbf{q} \boldsymbol{\sigma}_{2} \cdot \mathbf{q}}{q^{2}+M_{\pi}^{2}},
$$

with standard notations.

\section{Renormalization}

In EFT, all divergences appearing in loop diagrams are absorbed in parameters of the effective Lagrangian. While non-perturbative expressions may contain pieces which do not contribute in perturbative series, perturbative expansion of properly renormalized EFT amplitudes in the region of applicability of perturbation theory must reproduce the properly renormalized perturbative amplitudes.

\subsection{How not to renormalize in EFT}

To address the issue of non-perturbative renormalization in nuclear EFT we consider a simple case of the LS equation with a contact interaction potential

$$
V\left(p^{\prime}, p\right)=C+C_{2}\left(p^{\prime 2}+p^{2}\right) .
$$

The corresponding LS equation can be solved exactly leading to 3 .

$$
T=\frac{C+C_{2}^{2} \hbar I_{5}+k^{2} C_{2}\left(2-C_{2} \hbar I_{3}\right)}{\left(1-C_{2} \hbar I_{3}\right)^{2}-\left[C+C_{2}^{2} \hbar I_{5}+k^{2} C_{2}\left(2-C_{2} \hbar I_{3}\right)\right] \hbar I(k)},
$$

where $I(k), I_{3}$ and $I_{5}$ are divergent one-loop integrals. The parameters $C$ and $C_{2}$ can be determined by matching to the effective range expansion

$$
\Re\left(T^{-1}\right)=-\frac{m}{4 \pi}\left(-\frac{1}{a}+\frac{1}{2} r k^{2}+\ldots\right),
$$

yielding

$$
\begin{aligned}
C & =\frac{6 \pi^{2}\left[a^{2} \hbar \Lambda^{3} m(64 \hbar-3 \pi \Lambda r)-6\left(D-3 \pi^{2} \Lambda m\right)-62 \pi a \hbar \Lambda^{2} m\right]}{5 \hbar \Lambda^{2} m^{2}\left[a^{2} \hbar \Lambda^{2}(16 \hbar-\pi \Lambda r)-12 \pi a \hbar \Lambda+3 \pi^{2}\right]}, \\
C_{2} & =-\frac{6 \pi^{2}\left[-D+a^{2} \hbar m \Lambda^{3}(16 \hbar-\pi r \Lambda)-12 \pi a \hbar m \Lambda^{2}+3 \pi^{2} m \Lambda\right]}{\hbar m^{2} \Lambda^{4}\left[a^{2} \hbar \Lambda^{2}(16 \hbar-\pi r \Lambda)-12 \pi a \hbar \Lambda+3 \pi^{2}\right]},
\end{aligned}
$$

where

$$
D=\sqrt{3} \sqrt{\Lambda^{2} m^{2}(\pi-2 a \hbar \Lambda)^{2}\left(a^{2} \hbar \Lambda^{2}(16 \hbar-\pi \Lambda r)-12 \pi a \hbar \Lambda+3 \pi^{2}\right)} .
$$

Using these expressions the scattering amplitude can be expressed in terms of $a$ and $r$

$$
\begin{aligned}
T^{-1}= & \frac{m}{4 \pi^{2} a\left[a\left(\pi k^{2} r \Lambda-4 \hbar\left(k^{2}+\Lambda^{2}\right)\right)+2 \pi \Lambda\right]}\left\{2 \Lambda \left[a^{2} \hbar k^{2}(\pi r \Lambda-4 \hbar)-2 \pi a \hbar \Lambda\right.\right. \\
& \left.\left.+\pi^{2}\right]+a \hbar k \ln \frac{\Lambda-k}{\Lambda+k}\left[a\left(\pi k^{2} r \Lambda-4 \hbar\left(k^{2}+\Lambda^{2}\right)\right)+2 \pi \Lambda\right]\right\}+i \hbar \frac{m k}{4 \pi} .
\end{aligned}
$$


The amplitude $T^{-1}$ is finite for $\Lambda \rightarrow \infty$ resulting in

$$
T^{-1}=-\frac{m}{4 \pi}\left(-\frac{1}{a}+\frac{1}{2} r k^{2}-i \hbar k\right)+\mathcal{O}\left(\Lambda^{-1}\right) .
$$

However, in the loop expansion of the "renormalized" amplitude of Eq. (4)

$$
\begin{aligned}
T & =\frac{2 \pi a^{2} k^{2} r}{m}+\frac{4 \pi a}{m}-\frac{i \pi a^{2} \hbar k\left(a k^{2} r+2\right)^{2}}{m} \\
& +\hbar \frac{2 m k^{4}}{\pi^{2}}\left[-\frac{2 a^{4} k^{4} \Lambda r^{2}}{m}+\frac{2\left(a^{4} k^{6} r^{2}+4 a^{3} k^{4} r\right)}{\Lambda m}+\mathcal{O}\left(\Lambda^{-2}\right)\right]+\mathcal{O}\left(\hbar^{2}\right)
\end{aligned}
$$

we see that not all divergences are removed. Therefore, the above "non-perturbative renormalization", although it gives a finite result, is not an EFT renormalization. This procedure is more in spirit of "peratization" [5, 6. Not surprisingly, EFT using the "non-perturbative renormalization" fails to describe the phase shifts even at $\mathrm{N}^{3} \mathrm{LO}$ [7.

\section{Modified Weinberg's approach}

Equation (11) with the LO potential of Eq. (2) is not renormalizable, i.e. not all the divergences appearing in iterations of the LS equation can be absorbed into redefinition of parameters of $V_{0}$. This problem is often referred to as "inconsistency of Weinberg's approach" 4].

A modified, renormalizable version of Weinberg's approach has been proposed in Ref. 8]. We start with the manifestly Lorentz invariant effective Lagrangian of interacting pions and nucleons and use time ordered perturbation theory [9. The effective NN potential $V$ is defined as a sum of two-nucleon-irreducible diagrams. The off-shell nucleon-nucleon scattering amplitude $T$ satisfies the integral equation

$$
T=V+V G T
$$

where $G$ is the two-nucleon propagator. We expand $V, G$ and $T$ in small parameters,

$$
T=T_{0}+T_{1}+T_{2}+\cdots, \quad G=G_{0}+G_{1}+G_{2}+\cdots, \quad V=V_{0}+V_{1}+V_{2}+\cdots,
$$

and solve $T$ order by order. The LO amplitude is obtained by solving the equation

$$
T_{0}=V_{0}+V_{0} G_{0} T_{0} .
$$

Using $T_{0}$, the next-to-leading order (NLO) amplitude is obtained as

$$
T_{1}=V_{1}+T_{0} G_{0} V_{1}+V_{1} G_{0} T_{0}+T_{0} G_{0} V_{1} G_{0} T_{0}+T_{0} G_{1} T_{0} .
$$

Further, using $T_{0}$ and $T_{1}$, we calculate the NNLO amplitude $T_{2}$ etc.

The leading-order equation in the center-of-mass frame has the form

$$
T_{0}\left(\mathbf{p}^{\prime}, \mathbf{p}\right)=V_{0}\left(\mathbf{p}^{\prime}, \mathbf{p}\right)-\frac{m^{2}}{2} \int \frac{d^{3} \mathbf{k}}{(2 \pi)^{3}} \frac{V_{0}\left(\mathbf{p}^{\prime}, \mathbf{k}\right) T_{0}(\mathbf{k}, \mathbf{p})}{\left(\mathbf{k}^{2}+m^{2}\right)\left(p_{0}-\sqrt{\mathbf{k}^{2}+m^{2}}+i \epsilon\right)},
$$

where $p_{0}=\sqrt{\mathbf{p}^{2}+m^{2}}$ is the energy of a single nucleon. This equation was first obtained in Ref. [10]. Its iterations generate only overall logarithmic divergences. Therefore, the LO equation is perturbatively renormalizable. 
To investigate the non-perturbative regime, we notice that the ultraviolet behavior of Eq. (6) corresponds to the potential $\sim 1 / r^{2}$ for $r \rightarrow 0$ in $2+1$ dimensions. More singular $\sim 1 / r^{3}$ ultraviolet behavior in non-relativistic EFT is an artifact of that formulation. This can be easily understood for the cutoff-regularized one-loop integral

$$
I=\frac{4 i}{(2 \pi)^{4}} \int \frac{d^{4} k \theta(\Lambda-|\mathbf{k}|)}{\left[k^{2}-m^{2}+i 0^{+}\right]\left[(P-k)^{2}-m^{2}+i 0^{+}\right]},
$$

where $P=\left(2 \sqrt{m^{2}+\mathbf{p}^{2}}, \mathbf{0}\right)$. The result of the above integral for $\Lambda>|\mathbf{p}|$ reads:

$I=\frac{|\mathbf{p}|}{\pi^{2} \sqrt{m^{2}+\mathbf{p}^{2}}} \ln \frac{\Lambda \sqrt{m^{2}+\mathbf{p}^{2}}+|\mathbf{p}| \sqrt{\Lambda^{2}+m^{2}}}{m \sqrt{\Lambda^{2}-\mathbf{p}^{2}}}-\frac{\ln \frac{\Lambda+\sqrt{\Lambda^{2}+m^{2}}}{m}}{\pi^{2}}-\frac{i|\mathbf{p}|}{2 \pi \sqrt{m^{2}+\mathbf{p}^{2}}}$.

Expanding first in $1 / \Lambda$ and subsequently in $1 / m$ we obtain

$$
I=-\frac{i|\mathbf{p}|}{2 \pi m}-\frac{\ln \frac{\Lambda}{m}}{\pi^{2}}-\frac{\ln 2}{\pi^{2}}+\mathcal{O}\left(\frac{1}{\Lambda^{2}}, \frac{1}{m^{2}}\right) .
$$

On the other hand, expanding first in $1 / m$ and then in $1 / \Lambda$ leads to:

$$
I=-\frac{i|\mathbf{p}|}{2 \pi m}-\frac{\Lambda}{\pi^{2} m}+\frac{\mathbf{p}^{2}}{\pi^{2} \Lambda m}+\mathcal{O}\left(\frac{1}{m^{2}}, \frac{1}{\Lambda^{2}}\right) .
$$

The non-relativistic approach corresponds to the second expansion. It leads to the qualitatively different UV behavior. In perturbative calculations one compensates for this mismatch by readjusting terms from the effective non-relativistic Lagrangian 11. When re-summing iterations to all orders (e.g. by solving integral equations), one needs to include contributions of an infinite number of counter terms. Otherwise, one is not allowed to take $\Lambda>m$ in non-relativistic approach. On the other hand, the integral

$$
I_{1}=\frac{1}{(2 \pi)^{3}} \int \frac{d^{3} \mathbf{k} \theta(\Lambda-|\mathbf{k}|)}{\left[\mathbf{k}^{2}+m^{2}\right]\left[p_{0}-\sqrt{\mathbf{k}^{2}+m^{2}}+i 0^{+}\right]}
$$

corresponding to Eq. (6), has the proper UV behavior, guaranteeing the correct qualitative UV behavior in the modified Weinberg approach.

The leading-order partial wave equations are found to have unique solutions, except for the ${ }^{3} P_{0}$ wave. The equation in the ${ }^{3} P_{0}$ partial wave, similarly to the Skornyakov-TerMartirosyan equation [12, does not have an unique solution. Analogously to Ref. 13] we solve this problem by including a counter-term $\frac{c(\Lambda) p p^{\prime}}{\Lambda^{2}}$ at leading order.

The phase shifts obtained from the LO calculations are in a good agreement (within the accuracy expected at LO) with the Nijmegen PWA [16] (see figures).

The proper inclusion of the pion-exchange physics can also be tested in predictions for the coefficients of the effective range expansion

$$
p^{2 l+1} \cot \delta_{l}(p)=-\frac{1}{a}+\frac{1}{2} r p^{2}+v_{2} p^{4}+v_{3} p^{6}+v_{4} p^{8}+\ldots,
$$

where $a, r$ and $v_{i}$ denote the scattering length, effective range and shape parameters for the orbital angular momentum $l$. The long-range tail of the interaction imposes correlations between the coefficients in the effective range expansion [14]. These correlations may be regarded as low-energy theorems (LETs). In tables 1 and 2 the LETs in the KSW and modified Weinberg approaches are compared with the results of the 
Table 1 Coefficients in ERE of the ${ }^{1} S_{0}$ phase shifts

\begin{tabular}{llllll}
\hline${ }^{1} S_{0}$ partial wave & $a[\mathrm{fm}]$ & $r[\mathrm{fm}]$ & $v_{2}\left[\mathrm{fm}^{3}\right]$ & $v_{3}\left[\mathrm{fm}^{5}\right]$ & $v_{4}\left[\mathrm{fm}^{7}\right]$ \\
\hline NLO KSW & fit & fit & -3.3 & 18 & -108 \\
LO Weinberg & fit & 1.50 & -1.9 & $8.6(8)$ & $-37(10)$ \\
Nijmegen PWA & -23.7 & 2.67 & -0.5 & 4.0 & -20 \\
\hline
\end{tabular}

Table 2 Coefficients in ERE of the ${ }^{3} S_{1}$ phase shifts

\begin{tabular}{llllll}
\hline${ }^{3} S_{1}$ partial wave & $a[\mathrm{fm}]$ & $r[\mathrm{fm}]$ & $v_{2}\left[\mathrm{fm}^{3}\right]$ & $v_{3}\left[\mathrm{fm}^{5}\right]$ & $v_{4}\left[\mathrm{fm}^{7}\right]$ \\
\hline NLO KSW & fit & fit & -0.95 & 4.6 & -25 \\
LO Weinberg & fit & 1.60 & -0.05 & $0.8(1)$ & $-4(1)$ \\
Nijmegen PWA & 5.42 & 1.75 & 0.04 & 0.67 & -4.0 \\
\hline
\end{tabular}
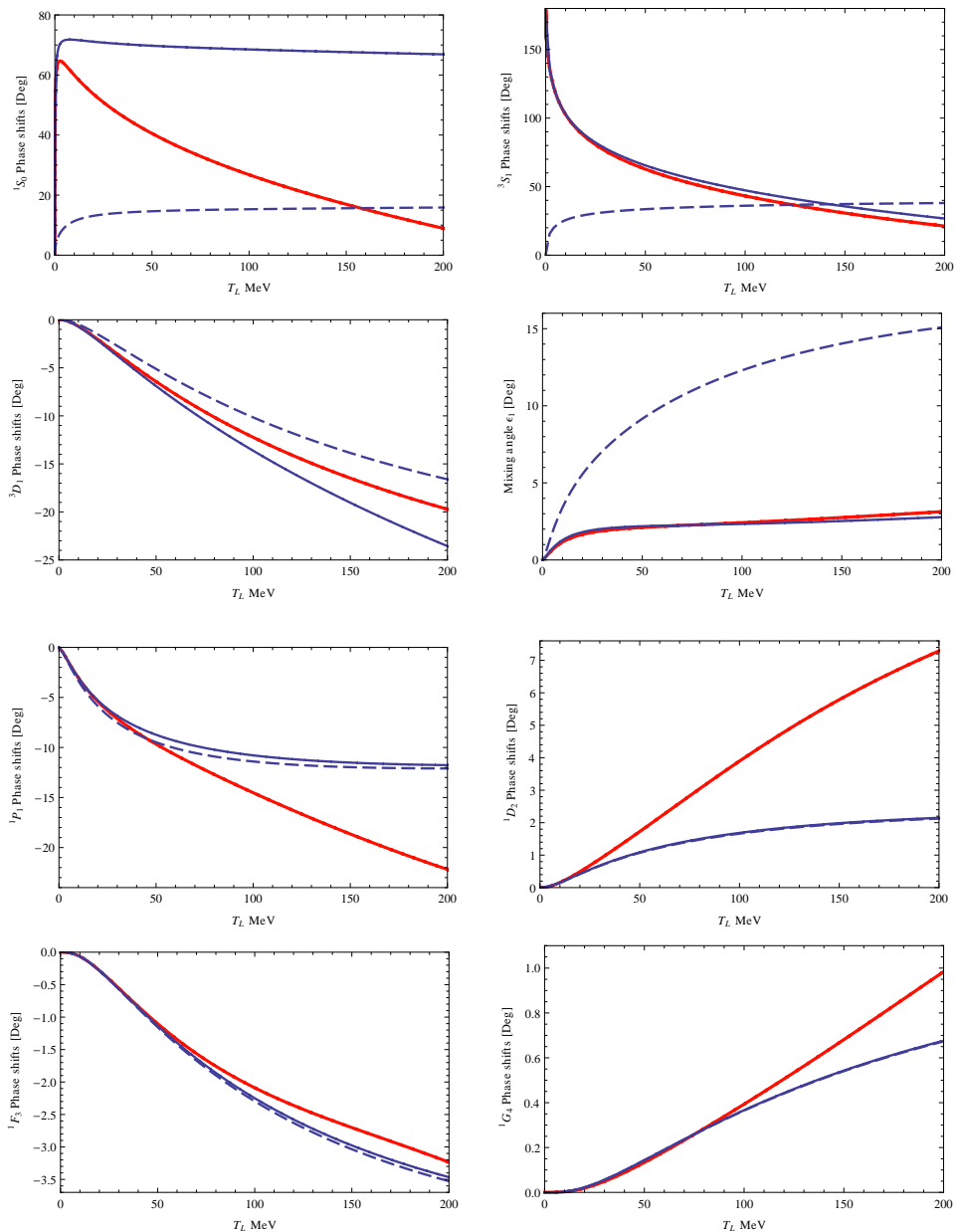

Fig. 1 Phase shifts at LO. The dashed and solid blue lines and the solid red line correspond to the Born approximation, the solution to the integral equation and the Nijmegen PWA, respectively. 

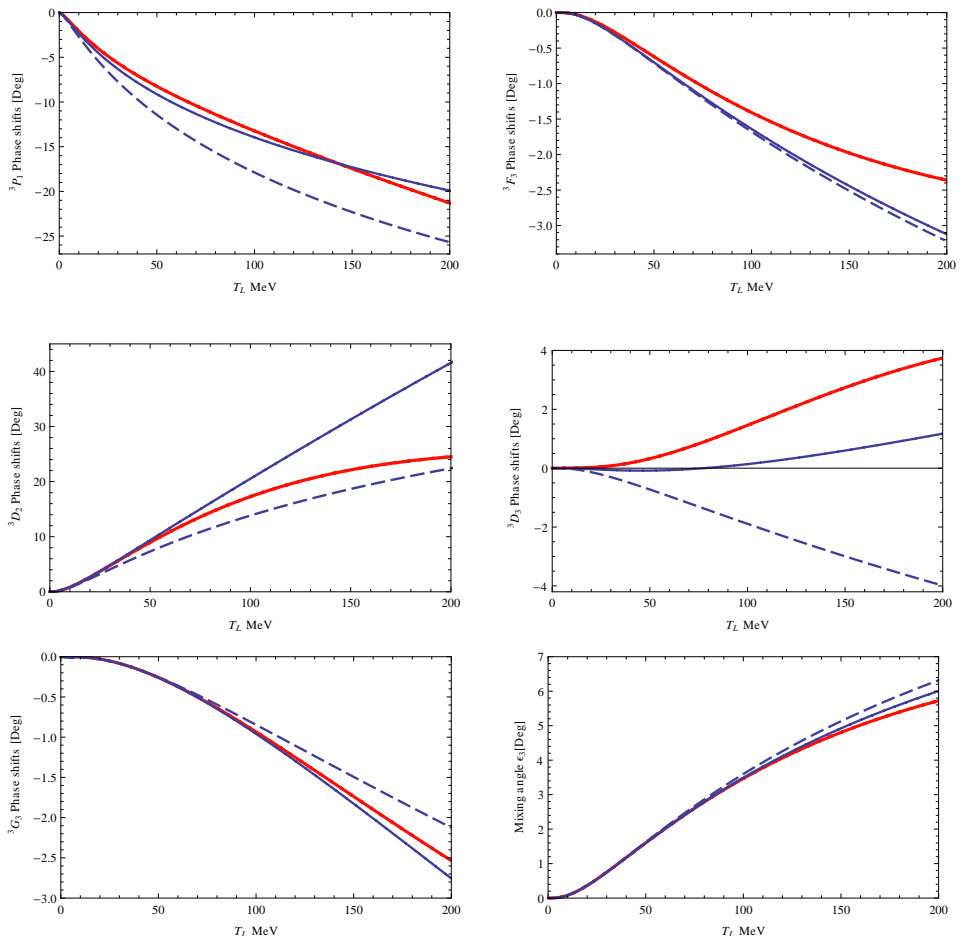

Fig. 2 Phase shifts at LO. The dashed and solid blue lines and the solid red line correspond to the Born approximation, the solution to the integral equation and the Nijmegen PWA, respectively.

Nijmegen PWA for the ${ }^{1} S_{0}$ and ${ }^{3} S_{1}$ partial waves, respectively. Coefficients corresponding to Nijmegen PWA are taken from Refs. [15], [16]. This work is supported by the EU (HadronPhysics3 project "Study of strongly interacting matter"), the European Research Council (ERC-2010-StG 259218 NuclearEFT) the DFG (GE 2218/2-1) and the Georgian Shota Rustaveli National Science Foundation (grant 11/31).

\section{References}

1. S. Weinberg, Phys. Lett. B 251, 288 (1990); Nucl. Phys. B363, 3 (1991).

2. E. Epelbaum, H. -W. Hammer and U.-G. Meißner, Rev. Mod. Phys. 81, 1773 (2009).

3. D. R. Phillips, S. R. Beane and T. D. Cohen, Annals Phys. 263, 255 (1998).

4. M. J. Savage, nucl-th/9804034

5. G. Feinberg and A. Pais, Phys. Rev. 131, 2724 (1963)

6. E. Epelbaum and J. Gegelia, Eur. Phys. J. A 41, 341 (2009).

7. C. Zeoli, R. Machleidt and D. R. Entem, arXiv:1208.2657] [nucl-th].

8. E. Epelbaum and J. Gegelia, Phys. Lett. B 716, 338 (2012).

9. S. Weinberg, Phys. Rev. 150, 1313 (1966).

10. V. G. Kadyshevsky, Nucl. Phys. B 6, 125 (1968).

11. J. Gegelia and G. Japaridze, Phys. Rev. D 60, 114038 (1999).

12. G. V. Skornyakov and Ter-Martirosyan, Sov. Phys. JETP 4, 648 (1957).

13. P. F. Bedaque, H. W. Hammer and U. van Kolck, Phys. Rev. Lett. 82, 463 (1999).

14. T. D. Cohen and J. M. Hansen, Phys. Rev. C 59, 13 (1999) nucl-th/9808038.

15. M. Pavon Valderrama, E. Ruiz Arriola, Phys. Rev. C 72, 044007 (2005).

16. J. J. de Swart, et al., nucl-th/9509032 